\title{
CT and MRI Evaluation of Bilateral Enlarged Vestibular Aqueduct Syndrome with Curved Reconstructions - A Case Report and Short Review of Literature
}

\author{
Chithra Ram ${ }^{1 *}$, Erik Seroogy ${ }^{2}$, Richard Sherry ${ }^{3}$ \\ ${ }^{1}$ Neuroradiologist, Associate Professor, Department of Radiology, University of Louisville, Kentucky, USA. \\ ${ }^{2}$ Medical student, University of Louisville, Kentucky, USA. \\ ${ }^{3}$ Neuroradiologist, RG Sherry MD PLLC, Watertown, New York, USA.
}

*Corresponding Author: Chithra Ram, Neuroradiologist, Assistant Professor, Department of Radiology, University of Louisville, Kentucky, USA.

Received date: May 27, 2021: Accepted date: June, 03 2021: Published date: July 19, 2021

Citation: Ram C., Seroogy E., Sherry R., (2021) CT and MRI Evaluation of Bilateral Enlarged Vestibular Aqueduct Syndrome with Curved Reconstructions - A Case Report and Short Review of Literature J.Clin Case Rep and Stu 2(3); DOI: 10.31579/2690-8808/079

Copyright: (C) 2021 Chithra Ram. This is an open access article distributed under the Creative Commons Attribution License, which permits unrestricted use, distribution, and reproduction in any medium, provided the original work is properly cited.

\begin{abstract}
Enlarged Vestibular Aqueduct Syndrome (EVAS) is a known, but rare entity. It represents a common cause of congenital sensorineural hearing loss, diagnosed more often in children with a slight female predominance. Herein, we report a case of bilateral Enlarged Vestibular Aqueduct Syndrome [EVAS] in a 26-year-old male. As it is a subtle finding on imaging, the interpreting radiologist must be aware of this entity to make the diagnosis. The diagnostic CT and MRI images of this patient are given along with curved CT and MRI reconstructions along the plane of the Vestibular Aqueduct [VA] extending to the endolymphatic sac [ES]. The CT reconstructions demonstrate the bony anatomy in great detail and the bilateral dilated VA. These help in excluding diseases like otospongiosis and inner ear anomalies. The curved MRI reconstruction CISS images through the VA demonstrate the entire endolymph channel. The reconstructed MRI Images clearly demonstrate a patent endolymph channel without any focal abnormality such as stricture, or other associated congenital anomalies.

Key words: dilated endolymphatic duct, dilated endolymphatic sac, dilated vestibular aqueduct syndrome, curved CT reformats of the vestibular aqueduct, curved MRI reformats of the vestibular aqueduct
\end{abstract}

\section{Introduction}

Enlarged Vestibular Aqueduct Syndrome (EVAS) is a known but rare entity. It is an important cause of sensorineural hearing loss. EVAS is diagnosed more frequently in children. The median age at diagnosis is 13 years. EVAS can be unilateral or bilateral. Bilateral involvement is more common than unilateral. EVAS ranges from complete deafness in infancy to reasonably well retained hearing in adulthood. Interestingly, the degree of aqueduct enlargement has not been correlated with the rate of attenuation of hearing in bilateral enlarged vestibular aqueduct syndrome (BEVAS). BEVAS is associated with mutations of SLC26A4, and these mutations correlate with the rate of hearing attenuation. Unilateral EVAS is not associated with SLC26A4, nor with any genetic syndromes. This condition can be congenital or acquired but is more commonly congenital.

\section{Case report}

26-year-old male was referred to the ENT clinic by his primary care physician for progressively worsening bilateral severe hearing loss. The patient had a childhood history significant for attention deficit disorder. Review of other systems was unremarkable. The patient was evaluated with audiogram, CT temporal bone, MRI of brain and IAC. Audiogram demonstrated bilateral down sloping which is associated with severe bilateral sensorineural hearing loss. Gene mapping for SLC26A4 mutations were not performed in this patient. Based on the findings, patient was scheduled to undergo cochlear implantation.

\section{Methods}

CT temporal bone without contrast was performed in a Somatom Definition Edge Siemens scanner [Siemens Medical Systems, USA]. Standard $0.6 \mathrm{~mm}$ axial CT bone algorithm images were obtained followed by $0.6 \mathrm{~mm}$ reconstructed images in coronal and oblique [Stenver's and 
Poschl's] planes. For the purpose of this case report, curved CT reformats were obtained from the vestibule through the vestibular aqueduct towards the endolymphatic sac.

MRI of the brain was performed on a 3T Siemens Spectra scanner [Siemens Medical Systems, Malvern, Pennsylvania, USA] using a standard head coil. Standard axial T1, axial T2, fat suppressed axial T2 FLAIR and GRE sequences without contrast were obtained at 22 FOV, 5 $\mathrm{mm}$ thickness and $1 \mathrm{~mm}$ spacing. Axial DWI and ADC brain pulse sequences were acquired without contrast using $28 \mathrm{FOV}, 5 \mathrm{~mm}$ thickness and 0 spacing. After intravenous administration of $20 \mathrm{ml}$ of Multihance [gadobenate dimeglumine, Bracco Diagnostics Inc., Monroe Township, NJ, USA], axial and coronal post contrast sequences were acquired using $5 \mathrm{~mm}$ thick slices with $1 \mathrm{~mm}$ spacing and $22 \mathrm{FOV}$.
MRI of the internal auditory canals was performed on a 3T Siemens Spectra scanner [Siemens Medical Systems, Malvern, Pennsylvania, USA] using a standard head coil. $3 \mathrm{~mm}$ Axial T1 and $1 \mathrm{~mm}$ axial CISS sequence were obtained pre contrast followed by post contrast $3 \mathrm{~mm}$ axial $\mathrm{T} 1$ and coronal T1 sequences. For the purpose of this case report, using CISS sequence, curved MRI reformats were obtained from the vestibule through the vestibular aqueduct to the endolymphatic sac.

\section{Imaging findings}

Nonenhanced temporal bone CT images show enlarged bilateral VA [Fig 1a].

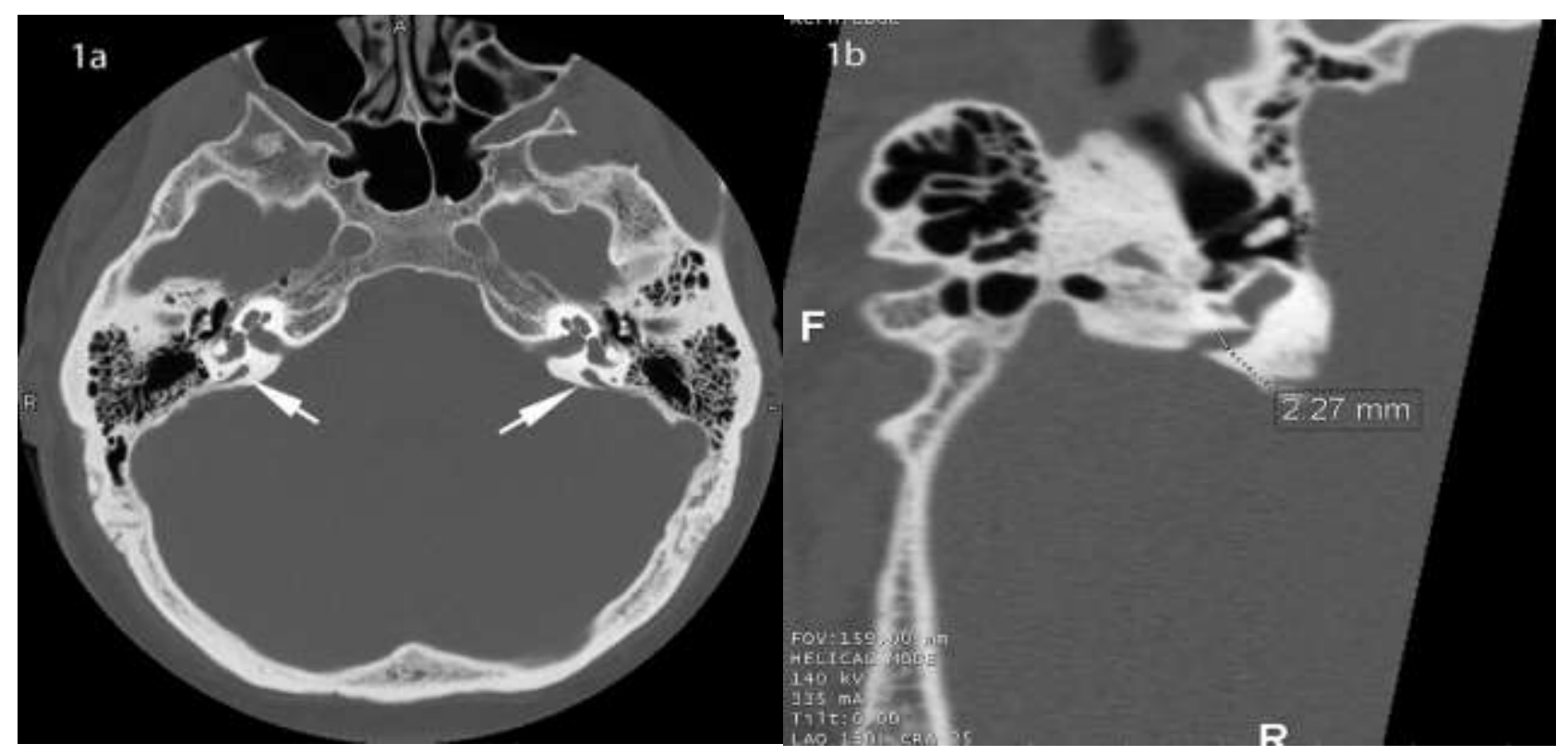

Figure 1. Non-enhanced CT Temporal bone axial image [1a] shows bilateral enlarged VA (arrows). The right VA measured 2.27 mm.

The VA are barely seen in a normal CT but in this patient, they are dilated and well seen. Right VA measures $2.27 \mathrm{~mm}$ [Fig.2b] and the left VA measures $2.3 \mathrm{~mm}$ perpendicular to the long axis of the VA.

Curved reconstructions were created by plotting along the VA [Fig 2a] in the nonenhanced temporal bone axial CT images. It shows enlarged right [Fig 2b] and left [Fig.2c] VA. The bony details are otherwise normal without any other co-existing inner ear congenital anomalies or bony changes such as otospongiosis.

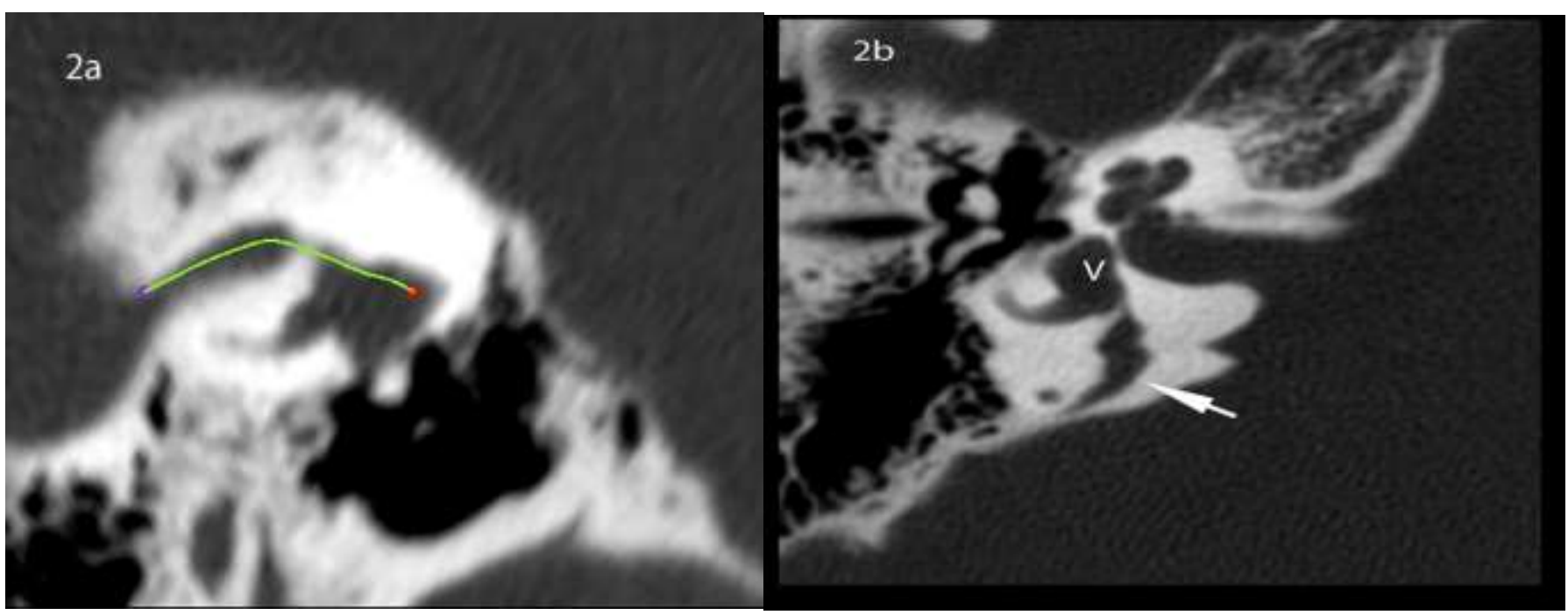




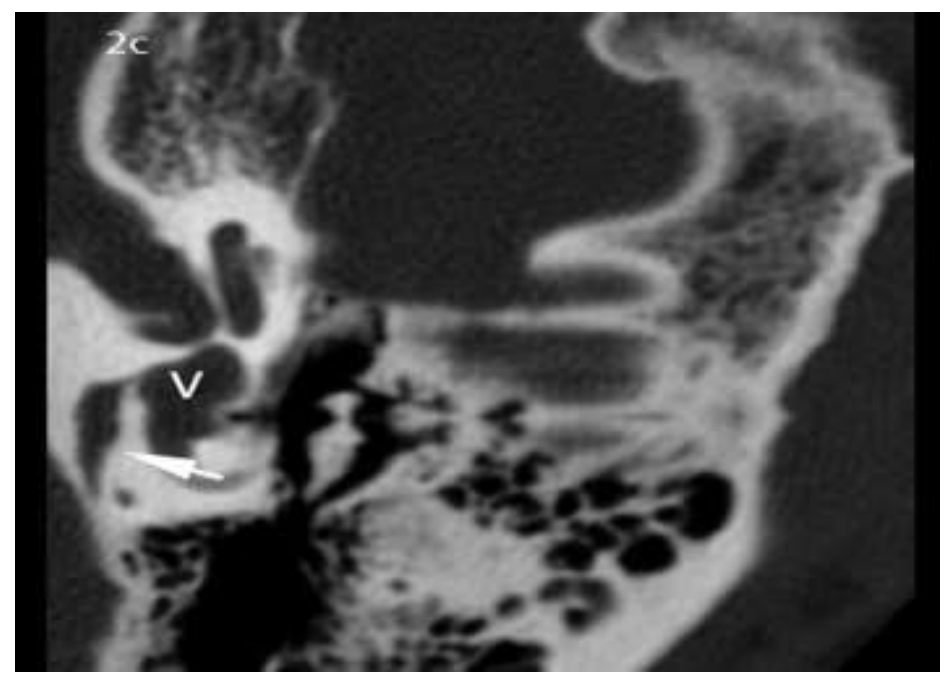

Figure 2. CT temporal bone image showing plane of curved reconstruction involving right vestibule and VA (2a). A similar plane of reconstruction was used on the left side. The curved reconstructed images of right VA (2b) and left VA [2c] from non-enhanced CT temporal bone demonstrates the widened VA (arrows).

MRI nonenhanced, enhanced and CISS images of the brain show normal brain parenchyma in the posterior fossa. Enlarged bilateral ES are seen but are better characterized in the dedicated MRI of the internal auditory canal [Fig 3a-c]. These images demonstrate differing signal intensities within the bilateral endolymphatic ducts and sacs, probably reflecting varying protein concentrations in the fluid on each side.

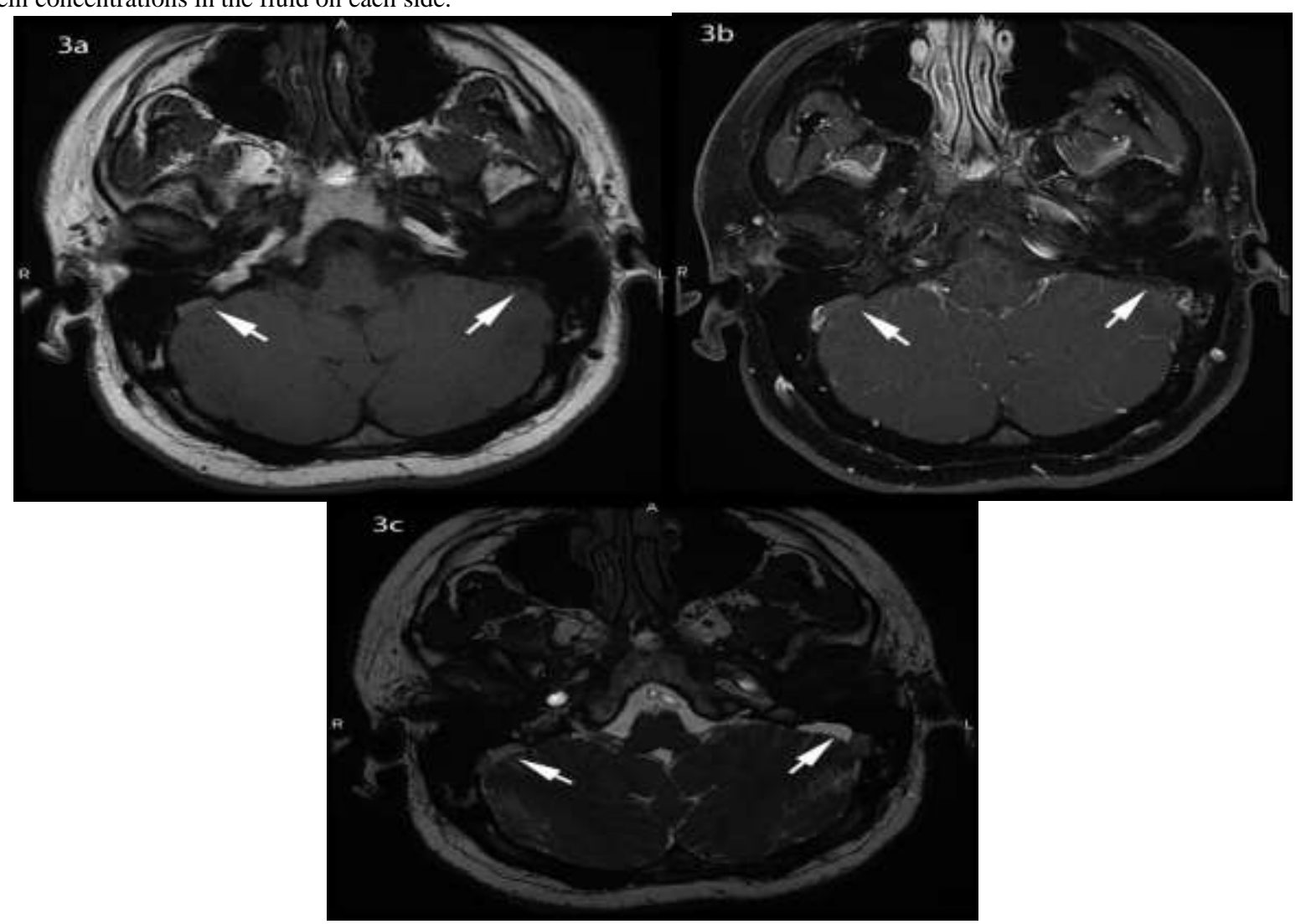

Figure 3. 3T Siemens Spectra scanner MRI Internal auditory canal protocol T1W image without contrast [3a], T1W image with contrast [3b] and $T 2 W$ CISS image [3c]. On all the images signal intensity in the ES (arrows) differs on the right and left. The right ES appears slightly hyperintense on T1W image and hypointense on T2W CISS image when compared to the left. These findings presumably are related to differing protein concentration in the two sides. No history of hemorrhage into the ES. No associated abnormal enhancement is seen bilaterally.

MRI nonenhanced and enhanced internal auditory canal images [Fig 3a, 3b] show enlarged bilateral ES and VA. 3D CISS is the most specific and sensitive MRI sequence for this diagnosis [Fig.3c]. Axial and curved reformat CISS images demonstrate the dilated endolymphatic duct and sac. 
Curved reconstructions of the endolymphatic duct and sac on both sides [Fig 4b-c] were obtained from the MRI 3D CISS images of the internal auditory canals. Figure 4a depicts the plane of curved reconstruction on CISS images on the left side. They show dilated ED and ES without focal abnormalities such as stricture within them. No other congenital anomalies were present.

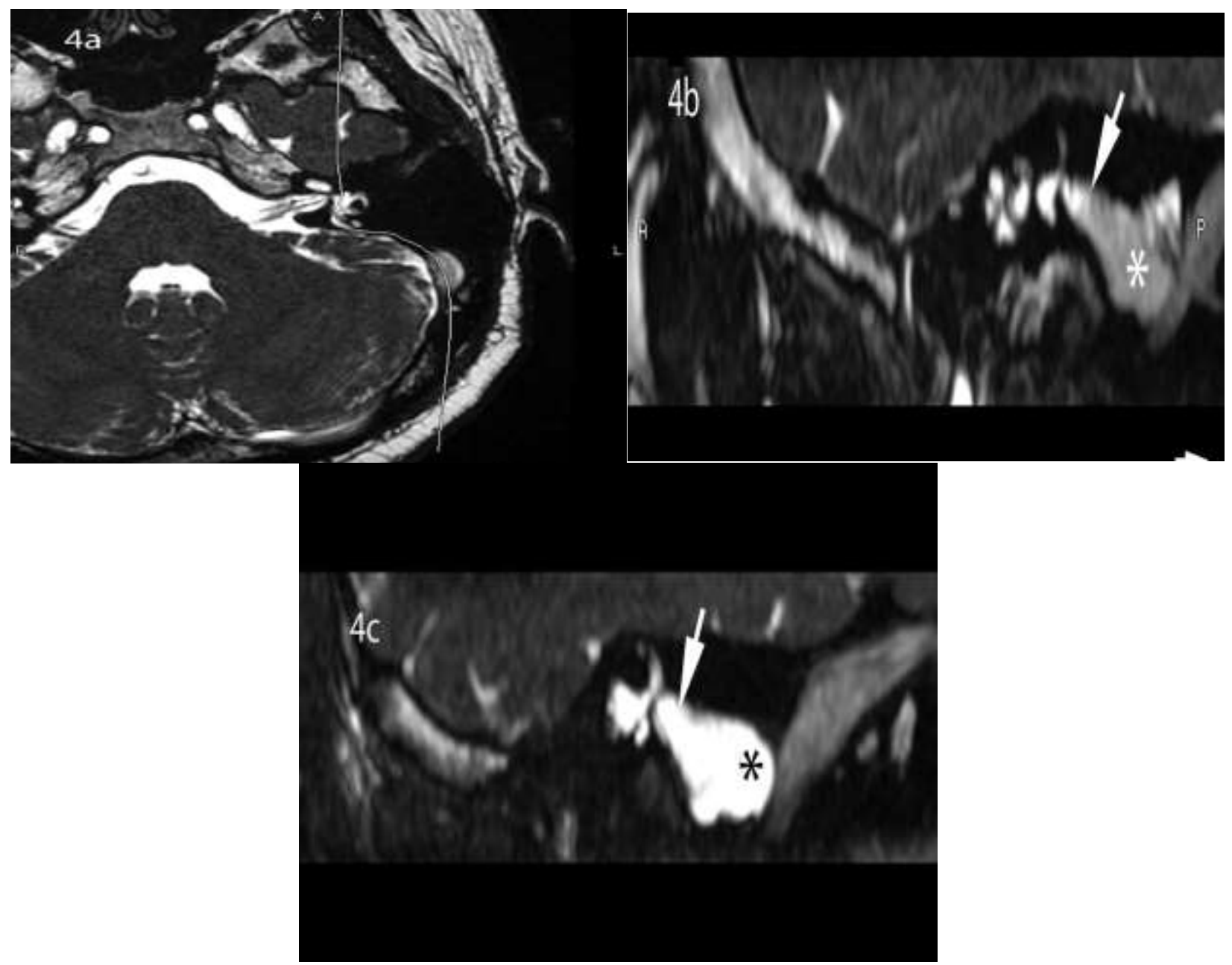

Figure 4. 3T Siemens Spectra scanner MRI axial T2 CISS image shows plane of curved reconstruction of CISS images from left vestibule to left endolymphatic sac (4a). A similar plane of reconstruction was used on the right side. The reformatted images of the right [4b] and left [4c] sides elegantly demonstrate the entire length of the ED on each side [arrows] and the enlarged ES [*]. There is no stricture or narrowing of the ED on either side. Note the disparity in signal intensity within the ED and ES on each side.

EVAS disproportionately affects children, representing a significant cause of pediatric sensorineural hearing loss. It comprises of $10-15 \%$ of all cases of sensorineural hearing loss $[1,2]$. The median age of diagnosis is 13 years. A slight female predominance has been suggested. [2] EVAS is bilateral in $90 \%$ of cases, with or without symmetry [3.] This condition is congenital in most cases, yet may also be acquired via head trauma, increased intracranial pressure, upper respiratory infection, or sudden pressure changes [4]. Moreover, a genetic component likely influences the disease process, as siblings are often co-inflicted, and the chance of familial presence may exceed $33 \%$. Using these results from their study, Tong et al. suggested a mixture of autosomal recessive and dominant inheritance patterns with incomplete penetrance/variable expression [5]. There is some controversy regarding EVA's origin, and two prevalent, competing hypotheses exist. One claims that EVA arises in early gestation due to arrested development, while the other states that EVA arises during later fetal/postnatal life from aberrant development $[6,7]$.

\section{Clinical presentation}

The vast majority of patients experience progressive and/or fluctuating bilateral onset of sensorineural hearing loss $[2,9]$. It is believed that the SLC26A4 mutations causing EVA causes hearing loss, rather than EVA directly resulting in hearing loss [8]. Vertigo and dizziness are typically present to some degree. EVA's clinical picture often mimics otosclerosis and other common etiologies of hearing loss when presenting in the adult population [9]. Further, about $60 \%$ of EVA patients also possess additional anatomic deficits, such as enlarged semicircular canals or hypoplastic cochlea, among others [10].

\section{Co-existing anomalies:}

The pathogenesis of EVA has been associated with inner ear anomalies and genetic syndromes in about $85 \%$ of patients, including Pendred syndrome, cochlear anomalies (i.e., cochlear hypoplasia, Mondini malformation, etc.), otofaciocervical syndrome, CHARGE syndrome, vestibular and semicircular canal anomalies, etc. The majority of these anomalies are linked to mutations in SLC26A4, which codes for pendrin, a cellular transporter of various negatively charged ions. Yet, polygenic influences from still-undefined genes may still play a role [5]. Pendred 
syndrome refers to EVA that is coupled with genetic thyroid dysfunction $[1,10]$.

\section{Pathophysiology}

The pathophysiological mechanism of hearing loss in EVA has not yet been fully elucidated. Back pressure of endolymph onto the cochlea, leak of hyperosmolar endolymph into the cochlea, and ion pump saturation leading to inner ear electrolyte imbalances are widely proposed models [10].

\section{Imaging findings}

EVAS displays enlarged VA on CT. The endolymphatic duct and sac usually are also enlarged and are better seen on MRI. Abnormalities involving the cochlea, vestibule and semicircular canals can be associated. Middle and external ear structures are usually within normal limits.

\section{Non-enhanced CT:}

In literature, multiple thresholds regarding the size of enlarged VA exist with some studies cautioning against the use of any specific threshold whatsoever. Statistically, at least 120 reference values are required to calculate any valid $95 \%$ mid-interval $\left(2.5^{\text {th }}-97.5^{\text {th }}\right.$ percentile $)$. Using 146 reference values, Vijayasekaran et al. calculated that VA measured $<0.9$ $\mathrm{mm}$ at midpoint and $<1.9 \mathrm{~mm}$ at the operculum, ideally measured halfway between the crus and the aperture on an axial view (i.e., AP dimension) [11]. As such, the vestibular aqueduct is considered enlarged if measuring $\geq 1 \mathrm{~mm}$ at the midpoint of the aqueduct and $\geq 1.9 \mathrm{~mm}$ at operculum, perpendicular to the long axis of the VA. In Poschl's plane (images obtained parallel to the superior semicircular canal) measurements $\geq 1.2$ $\mathrm{mm}$ at midpoint and $\geq 1.3 \mathrm{~mm}$ at operculum have been suggested [12]. Some studies suggest mid vestibular aqueduct measurement of $\geq 1.5 \mathrm{~mm}$ as enlarged [5]. The posterior margin of the petrous bone can be scalloped due to the enlarged ES..

\section{MRI:}

T1WI shows low to intermediate signal endolymphatic sac along the posterior petrous bone. 3D CISS [Siemens] or equivalent FIESTA [GE], is the most specific and sensitive sequence and it shows enlarged ES and duct with variable signal likely based on its proteinaceous content. Often, the typical high-signal fluid within the posterior duct is supplanted by low-signal fluid, owing to its proximity to loose or fibrous connective tissue. In over $80 \%$ of cases, additional inner ear structural abnormalities are also visualized, ranging from conspicuous dysplasia of inner ear structures to more subtle asymmetry $[13,14]$.

\section{Prognosis}

The degree of VA enlargement (ranging from $1.5 \mathrm{~mm}$ to $8 \mathrm{~mm}$ ) has not been correlated with the rate of attenuation of hearing in BEVAS. However, mutations of SLC26A4 have been shown to do so. [10] This rate ranges from complete deafness in infancy to decently retained hearing into adulthood. Prognosis is considered better for unilateral hearing loss and/or late onset hearing loss. While unilateral EVAS still holds a significant risk for hearing loss in the affected ear, this condition's etiology may be different altogether as it is not associated with SLC26A4, nor with the aforementioned genetic syndromes seen in BEVAS [8, 15]. Overall prognosis of EVAS is difficult to predict reliably.

\section{Treatment}

Therapeutic planning is often guided by accompanying inner ear abnormalities. First-line treatment of EVAS involves temporary hearing aids and rehabilitation followed by cochlear implantation. Cochlear implantation consistently yields profoundly improved outcomes in audiometric function, comparable to that of healthy patients [13]. The presence of incomplete partition of the cochlea or other concurrent inner ear anomalies may temper efficacy of implantation by up to a third, though function is still greatly improved [16]. Accordingly, this procedure represents the curative mainstay of EVAS. Intratympanic corticosteroid use has been studied, but the results remain controversial. Some studies report that $80-85 \%$ of their patients demonstrated hearing improvement with steroid therapy, but meta-analyses on the topic show no significant benefit. This may be due to the tendency for patients with mild EVAS cases to receive steroids, while those with severe progression generally require cochlear implantation [17]. Other previously attempted surgeries, such as endolymphatic sac obliteration or decompression shunting, are ineffective for EVAS and may even hasten its progressive hearing loss [18].

\section{Conclusion}

EVAS is a relatively rare entity, which can lead to sensorineural deafness. Though it's most commonly seen in childhood, with female preponderance, this is a case report in a 26-year-old male, underscoring the need to be alert to this diagnosis in adults. The CT and MRI images clearly demonstrate previously undiagnosed bilateral EVAS in this patient. Awareness of this subtle entity is essential for the interpreting radiologist in order to make a prompt and correct diagnosis. In this case, curved reconstructions through the entire VA extending to the ES were created using both CT images and thin volumetric axial CISS sequence. The reconstructed images clearly demonstrated the entire dilated channel of the VA, ED and ES. Focal lesions like distal widening, stricture or associated congenital anomalies can be more easily identified using these reconstructed images.

\section{Abbreviations}

EVAS - Enlarged Vestibular Aqueduct Syndrome, BEVAS - Bilateral Enlarged Vestibular Aqueduct Syndrome, VA - vestibular aqueduct, ED - endolymphatic duct, ES - endolymphatic sac, CT- Computerized Tomography, MRI- Magnetic resonance Imaging, 3D CISS - threedimensional constructive interference in steady state, FIESTA - Fast Imaging Employing Steady -state Acquisition.

\section{Conflict of interest}

None.

\section{Funding}

None.

\section{References}

1. Archibald HD, Ascha M, Gupta A, et al Hearing loss in bilateral enlarged vestibular aqueduct syndrome. International Journal of Pediatric Otorhinolaryngology x; 118: $147-151$.

2. Noguchi Y, Fukuda S, Fukushima K, et al. (2017). A nationwide study on enlargement of the vestibular aqueduct in Japan. Auris Nasus Larynx; 44: 33 - 39.

3. Joshi VM, Navlekar SK, Kishore GR, et al. (2012). CT and MR imaging of the inner ear and brain in children with congenital sensorineural hearing loss. Radiographics; 32(3): 683-698.

4. Swartz JD \& Mukherji SK. (2009). The inner ear and otodystrophies. In: Swartz JD, Loevner LA, eds. Imaging of the temporal bone. 4th ed. New York, NY: Thieme; 298-411.

5. Tong KA, Harnsberger HR, Dahlen RT, et al. (1997). Large vestibular aqueduct syndrome: a genetic disease? Am J Roentgenol; 168(4): 1097-1101.

6. Pyle M. (2000). Embryological development and large vestibular aqueduct syndrome. The Laryngoscope; 110 (11): 1837 - 1842.

7. Gopen Q, Zhou G, Whittemore K, \& Kenna M. (2011). Enlarged vestibular aqueduct: review of controversial aspects. The Laryngoscope; 121: 1971 - 1978. 
8. Berretini S, Forli F, Bogazzi F, et al. (2005). Large vestibular aqueduct syndrome: audiological, radiological, clinical, and genetic features. American Journal of Otolaryngology; 26: 363 - 371.

9. Wieczorek SS, Anderson ME, Harris DA, \& Mikulec AA. (2013). Enlarged vestibular aqueduct syndrome mimicking otosclerosis in adults. Am J Otolaryngol; 34(6): 619-625.

10. Okamoto K, Ito J, Furusawa T, et al. (1998). MRI of enlarged endolymphatic sacs in the large vestibular aqueduct syndrome. Neuroradiology; 40: 167 - 172.

11. Vijayasekaran S, Halsted MJ, Boston M, et al. (2007). When is the vestibular aqueduct enlarged? A statistical analysis of the normative distribution of vestibular aqueduct size. Am J Neuroradiol. 28(6): 1133-1138.

12. Naganawa S, Koshikawa T, Iwayama E, et al. (2000) MR imaging of the enlarged endolymphatic duct and sac syndrome by use of a 3D fast asymmetric spin-echo sequence: volume and signal-intensity measurement of the endolymphatic duct and sac and area measurement of the cochlear modiolus. Am J Neuroradiol; 21(9): 1664-1669.
13. Mafee MF, Charletta D, Kumar A, \& Belmont H. (1992). Large vestibular aqueduct and congenital sensorineural hearing loss. Am J Neuroradiol; 13(2): 805-819.

14. Davidson HC, Harnsberger HR, Lemmerling MM, et al. (1999). MR evaluation of vestibulocochlear anomalies associated with large endolymphatic duct and sac. Am J Neuroradiol; 20(8): 1435-1441.

15. Greinwald J, deAlarcon A, Cohen A, et al. (2013) Significance of unilateral enlarged vestibular aqueduct. The Laryngoscope; 123: 1537 - 1546.

16. Manzoor NF, Wick CC, Wahba M, et al. (2016). Bilateral sequential cochlear implantation in patients with enlarged vestibular aqueduct (EVA) syndrome. Otology \& Neurotology; 37: e96 - e103.

17. Patel ND, Ascha MS, Manzoor NF, et al. (2018). Morphology and cochlear implantation in enlarged vestibular aqueduct. $A m J$ Otolaryngol; 39(6): 657-663.

18. Grimmer JF, Hedlund G, \& Park A. (2008). Steroid treatment of hearing loss in enlarged vestibular aqueduct anomaly. International Journal of Pediatric Otorhinolaryngology; 72(11): 1711-1715.
This work is licensed under Creative

Commons Attribution 4.0 License

To Submit Your Article Click Here: Submit Manuscript

DOI: $10.31579 / 2690-8808 / 079$

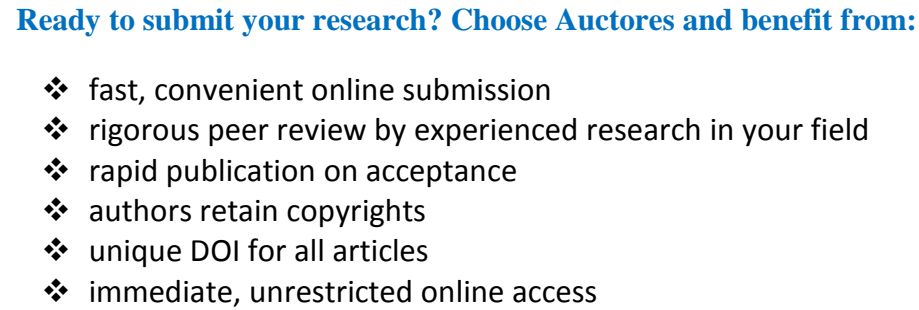

At Auctores, research is always in progress.

Learn more www.auctoresonline.org/journals/journal-of-clinical-casereports-and-studies 